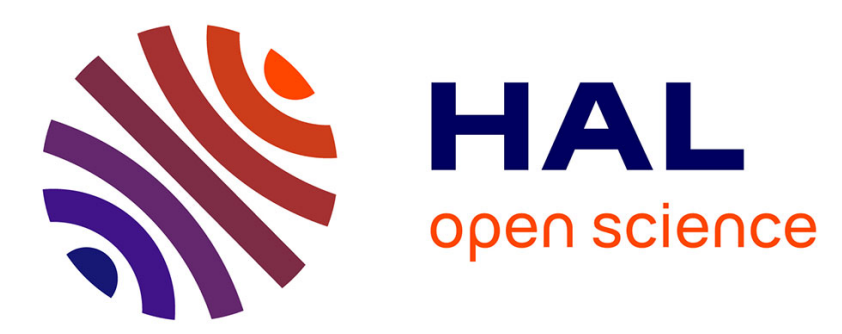

\title{
Experimental full wavefield reconstruction and band diagram analysis in a single-phase phononic plate with internal resonators
}

\author{
N. Kherraz, M. Radzieński, M. Mazzotti, P. Kudela, F. Bosia, A. S. Gliozzi, \\ D. Misseroni, N. M. Pugno, W. Ostachowicz, M. Miniaci
}

\section{To cite this version:}

N. Kherraz, M. Radzieński, M. Mazzotti, P. Kudela, F. Bosia, et al.. Experimental full wavefield reconstruction and band diagram analysis in a single-phase phononic plate with internal resonators. Journal of Sound and Vibration, 2021, 503, pp.116098. 10.1016/j.jsv.2021.116098 . hal-03440709

\author{
HAL Id: hal-03440709 \\ https://hal.science/hal-03440709
}

Submitted on 22 Nov 2021

HAL is a multi-disciplinary open access archive for the deposit and dissemination of scientific research documents, whether they are published or not. The documents may come from teaching and research institutions in France or abroad, or from public or private research centers.
L'archive ouverte pluridisciplinaire $\mathbf{H A L}$, est destinée au dépôt et à la diffusion de documents scientifiques de niveau recherche, publiés ou non, émanant des établissements d'enseignement et de recherche français ou étrangers, des laboratoires publics ou privés. 


\title{
Experimental full wavefield reconstruction and band diagram analysis in a single-phase phononic plate with internal resonators
}

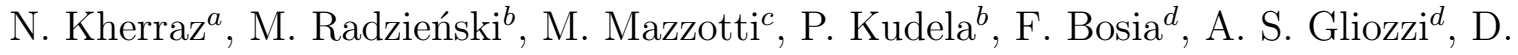

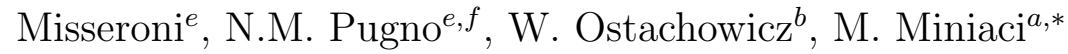 \\ a) CNRS, Univ. Lille, Ecole Centrale, ISEN, Univ. Valenciennes, IEMN - UMR 8520, 59046, Lille cedex, \\ France \\ b) Institute of Fluid-Flow Machinery, Polish Academy of Science, Fiszera 14 st. 80-231 Gdańsk, Poland \\ c) Department of Mechanical Engineering, CU Boulder, 1111 Engineering Drive, UCB 427 Boulder, CO \\ 80309, USA \\ d) Department of Applied Science and Technology, Politecnico di Torino, Corso Duca degli Abruzzi 24, \\ 10124, Torino, Italy \\ e) Laboratory of Bio-Inspired, Bionic, Nano, Meta Materials \& Mechanics, Department of Civil, \\ Environmental and Mechanical Engineering, University of Trento, Via Mesiano77, 38123 Trento, Italy \\ f) Queen Mary University of London, School of Engineering, Materials Science, Mile End Road, London \\ E1 $4 N S, U K$
}

\section{Abstract}

Research on phononic crystal architectures has produced many interesting designs in the past years, with useful wave manipulation properties. However, not all of the proposed designs can lead to convenient realizations for practical applications, and only a limited number of them have actually been tested experimentally to verify numerical estimations and demonstrate their feasibility.

In this work, we propose a combined numerical-experimental procedure to characterize the dynamic behavior of metamaterials, starting from a simplified 2D design to a real 3D manufacturing structure. To do this, we consider a new simplified design of a resonator-type geometry for a phononic crystal, and verify its wave filtering properties in wave propagation experiments. The proposed geometry exploits a circular distribution of cavities in a homogeneous material, leading to a central resonator surrounded by thin ligaments and an external matrix. Parametric simulations are performed to determine the optimal thickness of this design leading to a large full band gap in the $\mathrm{kHz}$ range. Full field experimental characterization of the resulting phononic crystal using a scanning laser Doppler vibrometer is then performed, showing excellent agreement with numerically predicted band gap prop-

* correspondence to: Marco Miniaci, tel +39 3292259 031, email: marco.miniaci@univ-lille.fr Preprint submitted to Elsevier 
erties and with their resulting effects on propagating waves. The outlined procedure can serve as a useful step towards a standardization of metamaterial development and validation procedures.

Keywords: Phononic Crystals, Elastic Metamaterials, Elastic Wave Propagation, Experimental Full Wavefield Reconstruction, Wavenumber-Frequency Analysis

\section{Introduction}

The investigation of elastic wave propagation phenomena in artificially structured composite materials is an active research topic in the scientific community. Shortly after the introduction of photonic crystals and electromagnetic metamaterials, their elastic counterpart, i.e., phononic crystals (PCs) and elastic metamaterials [1-3], have attracted increasing attention due to the possibility of reproducing in elasticity an abundant set of unusual physical properties [4], such as stop-band filtering [5, 6], negative refraction [7-9], acoustic lensing [10], ordinary [11, 12] and topologically protected [13-17] wave localization / splitting, and fluid elasticity [18]. Among these, the ability to attenuate elastic waves over entire frequency ranges, often referred to as phononic band gaps (BGs), is among the most attractive and studied properties. BGs occur due to three main mechanism: Bragg scattering, local resonance and inertial amplification [19-27].

Due to this property, phononic plates received great attention because of their potential for technological applications: structural health monitoring [28, 29], wave switching [30] and demultiplexing [31], micro-electro-mechanical systems [32, 32], cloaking [33], to cite a few. Among the possible configurations, phononic plates made of single or multiple constituents have been considered, including periodic distributions of inclusions, pillars / gratings on the plate surfaces, and empty holes [34].

In multi-material phononic plates, the shape, material type as well as the orientation of the inclusions strongly influence the existence and location in frequency of the BGs. The possibility to open both Bragg and locally resonant BG types was reported [35-37]. In single phase phononic crystals, it was shown that the local resonance of the pillars / inclusions was the dominant mechanism to open / shift BGs $[38,39]$. Plates with a periodic grating on the surface have also been investigated, and a relationship established between the width of the 
BG and the depth of the grooves [40]. While these two approaches inevitably lead to some geometrical / manufacturing complexity, phononic plates realized by through-the-thickness cavities in a homogeneous material remain a good compromise between a simpler fabrication procedure and good wave attenuation performance. Whilst numerical / theoretical works dealing with cavities perpendicular to the wave propagation plane are numerous, experimental measurements are often limited to few measurement points or small scanning regions. Our aim in this paper is thus to propose an in-depth numerical and experimental characterization procedure to validate metamaterial designs and develop them into functioning realistic structures. Inspired by the 2D geometry proposed for the first time by Bigoni and coworkers [10], here, we first investigate the influence of extending the design into a 3D realistic single-phase phononic plate with internal resonators generated by symmetrically arranged cavities, and then provide experimental evidence of a complete $\mathrm{BG}$ in the $\mathrm{kHz}$ frequency range. Full wavefield reconstruction of the wave propagation phenomena and a band diagram analysis in the wavenumber-frequency domain is provided and compared to numerical calculations.

\section{Design of the phononic plate}

\subsection{Eigenvalue problem}

In this section, we numerically investigate the dispersion properties of a periodic structure consisting of an inertial resonator embedded in a matrix through 8 ligaments, as shown in Fig. 1A. The structure is obtained by milling 8 cavities arranged in an octagonal pattern in a homogeneous Polymethyl methacrylate (PMMA, Perspex Black from Bayer) block, which divides the cell into three regions, named matrix, ligaments and resonator, respectively. This arrangement of material and cavities represents a good alternative to multi-phase resonators often made of a heavy core (in steel, tungsten or similar heavy metals) surrounded by a soft core (rubber, for instance) and embedded in an external matrix (often a polymer) [3]. In our case, the ligaments play the role of the soft coating.

In-plane geometrical parameters of the unit cell are given as a function of the ligament thickness $t=1 \mathrm{~mm}$ as follows: $A=19 \cdot t=19 \mathrm{~mm}, R_{e}=9 \cdot t, R_{i}=4 \cdot t$, as illustrated in Fig. 1A. These parameters have been chosen with specimen fabrication in mind (i.e., with the technical limitations of the milling process in mind). The density of PMMA is $\rho=1180$ 
$\mathrm{kg} / \mathrm{m}^{3}$ and the longitudinal and shear wave velocities are $c_{L}=2665 \mathrm{~m} / \mathrm{s}$ and $c_{T}=1363 \mathrm{~m} / \mathrm{s}$, respectively.

As a first step, the band structures are computed considering an infinitely duplicated unit cell in a periodic square array, and considering elastic wave propagation in the linear elastic regime (under the hypothesis of small displacements). The unit cell domain is meshed by means of 8-node hexagonal elements of maximum size $L_{F E}=0.1 \mathrm{~mm}$, which is found to provide accurate eigensolutions up to the frequency of interest [41]. Therefore, the resulting eigenvalue problem $\left(\mathbf{K}-\omega^{2} \mathbf{M}\right) \mathbf{u}=\mathbf{0}$ is solved by varying the non-dimensional wavevector $\mathbf{k}^{*}$ along the irreducible path $[M-\Gamma-X-M]$, with $M \equiv(\pi / A, \pi / A), \Gamma \equiv(0,0)$ and $X \equiv(\pi / A, 0)$ (see Fig. $1 \mathrm{~B})$, being $A$ the lattice parameter, namely the unit cell side.

The corresponding band diagrams are presented in Fig. 2A for different height to the lattice parameter ratios $H / A=[0.1,0.5,0.8,1.0,1.2]$. The dispersion curves are color coded according to the height $H$ of the unit cell. Specifically, the color bar of Fig. 2A varies gradually from dark blue (very thin unit cells) to dark red (thicker ones). The influence of the unit cell height on the dispersion curves is clearly visible from the diagrams. When an extremely flexible unit cell in the out-of-plane direction is considered (very small $H / A$ ratio, for instance 0.1 ), no complete $B G$ is visible in the diagram. This is due to a very low stiffness of the unit cell with respect to out-of-plane deformations, implying a large number of dispersion branches in the $[0-70] \mathrm{kHz}$ frequency range. When the height to the lattice parameter ratio $H / A$ increases, the structure gains stiffness against out-of-plane deformations and some of the previous modes migrate to higher frequencies. As a consequence, fewer curves are visible in the diagram in the same frequency range (compare for instance $H / A=0.1$ to $H / A=0.5$ ). In addition, specific modes (reported in Fig. 2B, C and highlighted in Fig. 2A by black arrows), undergo an opposite shift to higher / lower frequencies. This allows to open a BG of up to $8 \mathrm{kHz}$, achieved when $H / A=1$, and ranging approximately from 45 to $53 \mathrm{kHz}$. To gain a full understandings of the governing mechanism of the BG opening, we computed the imaginary part of the wavenumber, $\Im k(\omega)$, in the vicinity of the $B G$ frequencies. The so-called $k(\omega)$ method has been adopted [42]. Figure $3 A$ presents the imaginary part of the wavenumber, $\Im k(\omega)$, of the dispersion curves along the $\Gamma-X$ path for the $H / A=1$ case, in the $40-60 \mathrm{kHz}$ frequency range. Examining the curves exhibiting the lowest values of $\Im k(\omega)$ 
in proximity of the $B G$ frequency (those indicated by the black arrows), it is possible to notice the presence of both a parabolic and a spike-like behavior (Fig. 3B), both contributing to the $B G$ opening. This clearly proves that the considered structure opens a hybrid BG based on the interaction of both Bragg and local resonance mechanisms. If the ratio $H / A$ increases above unity, additional bands are introduced again in the $[0-70] \mathrm{kHz}$ frequency range reducing the BG width (see for instance the flexural mode reported in Fig. 2D).

\subsection{Numerical and experimental time-transient analysis on the finite structure}

In this section, a numerical time transient analysis on a finite structure is performed, and compared to experimental measurements, as schematically indicated in Fig. 4. In view of the experimental phase, a PMMA rectangular plate of length $4 \cdot L 1=1000 \mathrm{~mm}$, width $2 \cdot L 1=500$ mm and height $H=A=19 \mathrm{~mm}$ is considered. PMMA has been chosen as the material composing both the matrix and the inertial resonators because of wide availability and the possibility of manufacturing it with standard tools such as a milling machine. A PC region made of 200 unit cells such as the one reported in Fig. 1A disposed in the shape of square rings is introduced on the right side of the plate, as shown in Fig. 4A. In particular, the unit cells are distributed over a square frame of external and internal widths of $15 A$ and $5 A$. An unaltered area of $5 A \times 5 A=95 \times 95 \mathrm{~mm}^{2}$ is therefore included in the center of the phononic region. The sample used for the experimental analysis is manufactured by exporting the geometry from the finite element model, and importing it to the milling machine (EGX-600 Engraving Machine) software. The manufacturing process required a tolerance of $0.01 \mathrm{~mm}$ which is expected to have limited impact on the measurements.

Elastic guided waves are excited in correspondence of the point $E 1$ by means of a ceramic piezoelectric disk of $10 \mathrm{~mm}$ diameter bonded to the surface of the sample [43]. The plate has been suspended vertically through wires (as schematically reported in Fig. A1 of the Appendix A). This allowed us to prevent the sample from being subjected to bending, and to mimic the free boundary conditions implemented in the calculations. Also, we verified that the stress induced in the unit cells by such an experimental configuration (due to the only weight of the specimen itself) does not produce any measurable effect on the dispersion band diagram. To achieve BG alteration, much higher levels of stress are required [25], even in the case of extremely compliant designs [44]. 
As the first step, a pulse made of 2 sine cycles centered at $50 \mathrm{kHz}$ and modulated by a Hann window is fed to the function generator. This signal has been chosen so as to generate elastic waves with a much larger frequency content compared to the [45 - 53] $\mathrm{kHz}$ frequency range of the BG highlighted in Fig. 2A. The aim is to emphasize and quantitatively evaluate the screening power of the phononic region. Out-of-plane velocity is acquired through a PSV 400 3D scanning laser Doppler vibrometer by Polytec at the two acquisition points named $O 1$ and $O 2$ (Fig. 4A), taken at the same distance from the excitation point $E 1$, and chosen outside and inside the phononic region of the waveguide, respectively. In both cases, $3 \mathrm{~ms}$ long signals are recorded in order to allow multiple wave reflections to take place at both the edges of the waveguide, so as to allow elastic waves to impinge on the phononic region from multiple angles. After acquisition, signals are Fourier transformed and reported in Fig. 4B in order to highlight the differences between the two responses in terms of frequency content. The Fourier spectrum of the signal acquired outside the phononic region shows good levels of transmission within the excited frequency range $(30-90 \mathrm{kHz})$, whereas the signal recorded inside the phononic region (red markers) displays a clear amplitude drop in the BG region $(45-53 \mathrm{kHz})$. This is in agreement with the dispersion diagram presented in Fig. $2 \mathrm{~A}$ and clearly confirms the possibility of the waveguide to filter waves over the $[45-53] \mathrm{kHz}$ frequency range.

To gain further insights, full wave field reconstructions of the wave propagation phenomena over the orange rectangular area shown in Fig. 4A are performed and compared to numerical calculations. In the numerical model, elastic waves are excited by means of an out-of-plane imposed displacement (of amplitude $1 \times 10^{-6} \mathrm{~mm}$ ). At this stage, in addition to the previously described excitation, another pulse made of 21 sine cycles centered at 50 $\mathrm{kHz}$ and modulated by a Hann window is used as the excitation signal fed to the function generator (and as the imposed displacement in the numerical model). In both cases, the spatial scanning grid (orange rectangle in Fig. 4A) covers a $580 \times 500 \mathrm{~mm}^{2}$ of the right part of the phononic plate and consists of $293 \times 251$ equally spaced grid points. A total of 10 time averages were performed at each node to increase the signal to noise ratio. The knowledge of the velocity time histories at all grid points allows for the reconstruction of the time-evolving wavefields established in the scanning domain. Figures 4C,D show the numerical (left panels) 
and experimental (right panels) full wavefield reconstructions of the out-of-plane velocity for the Hann windowed excitation signals using 2 (Fig. 4C) and 21 (Fig. 4D) sine cycles centered at $50 \mathrm{kHz}$ fed in $E 1$. The out-of plane velocities are normalized with respect to the respective maximum amplitudes. When operating with elastic waves with a broadband energy content, the laser measures transmission inside the phononic region, allowing the wavefield reconstruction at a comparable intensity scale with respect to points of the plate not enclosed by the phononic region. However, unit cells scatter the wave field, resulting in an observable delay in the wave propagation. In this case, despite the scattering, the phononic region does not cause significant attenuation of the wave field. On the contrary, when observing the propagation of an elastic wave with a narrowband energy content totally falling inside the BG, strong destructive interferences due to the Bragg scattering are visible within the phononic region, clearly showing that waves are reflected between the transducer and the lower edge of the unit cell ring. This behavior is accompanied by an extremely low transmission due to the absence of detectable wave amplitudes inside the phononic region.

As a final experiment, elastic guided waves are excited in correspondence of the point E2. Among several types of excitation (larger number of cycles, other waveform shapes [triangular-like, chirp-like], central frequency), the function generator has been fed with a pulse made of 2 sine cycles centered at $40 \mathrm{kHz}$ and modulated by a Hann window, which showed to better inject energy in the system for the considered frequencies (also outside the $B G)$.

Out-of-plane velocity is measured along 647 equally spaced points (red dashed line reported in Fig. 4A). Measurements are plotted as a function of the scanning position along the scan line (x-axis) and time (y-axis) in Fig. 5A, where straight red lines denote the beginning and the end of the periodic region. Several reflections due to the impedance mismatch are clearly visible. Signals are then 2D-Fourier transformed and reported in Fig. 5B as an intensity plot of shades of grey normalized to its maximum value. Numerical dispersion curves are superimposed to this graph for the purpose of comparison [28, 45]. Due to the type of experimental set-up, mainly out-of plane modes are expected to be excited. Therefore, in-plane and out-of-plane polarization of the numerical modes are identified through the definition of a polarization factor $p=\frac{\int_{V}\left(u_{x}\right)^{2} d V}{\int_{V}\left[\left(u_{x}\right)^{2}+\left(u_{y}\right)^{2}+\left(u_{z}\right)^{2}\right] d V}$ for the unit cell, being $V$ the volume of the 
unit cell, $u_{x}, u_{y}$ and $u_{z}$ the displacement components along $x, y$ and $z$ axes, respectively. As a consequence, dispersion curves presented in Fig. 5B are shaded accordingly varying gradually from 0 (blue) to 1 (green). Thus, colors close to green indicate vibration modes that are dominantly polarized out-of-plane, while those close to blue are predominantly polarized in-plane. A very good agreement of the two maps is found, confirming that, due to the type of experimental set-up, mainly out-of plane modes are excited.

\section{Conclusions}

In this paper, we have presented a combined numerical and experimental characterization procedure to validate metamaterial designs to create realistic functional wave-filtering structures. We have considered an optimized design with respect to the plate thickness for a phononic crystal characterized by full BGs in the $\mathrm{kHz}$ range, and fully demonstrated its efficiency in wave propagation experiments. The design itself can be useful addition to other architectures considered in the literature presenting wide BGs, with the additional advantage of a simple fabrication process, e.g. by milling. More importantly, the presented experimental characterization procedure can serve as a general method for standardized testing and evaluation of phononic crystal designs. To the best of our knowledge, this is the first work to provide full experimental characterization for this type of geometry.

\section{Acknowledgments}

This project has received funding from the European Union's Horizon 2020 research and innovation programme under grant agreement No. 863179.

\section{Appendix A}

In this Appendix a schematic representation of how the plate was suspended during the experimental phase is provided in Fig. A1. 


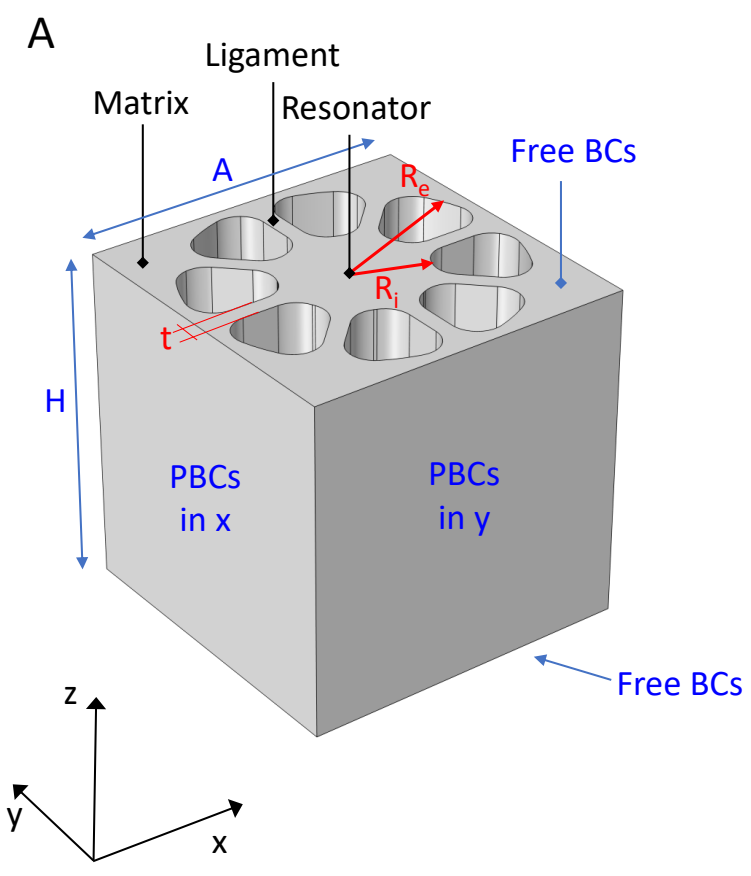

B

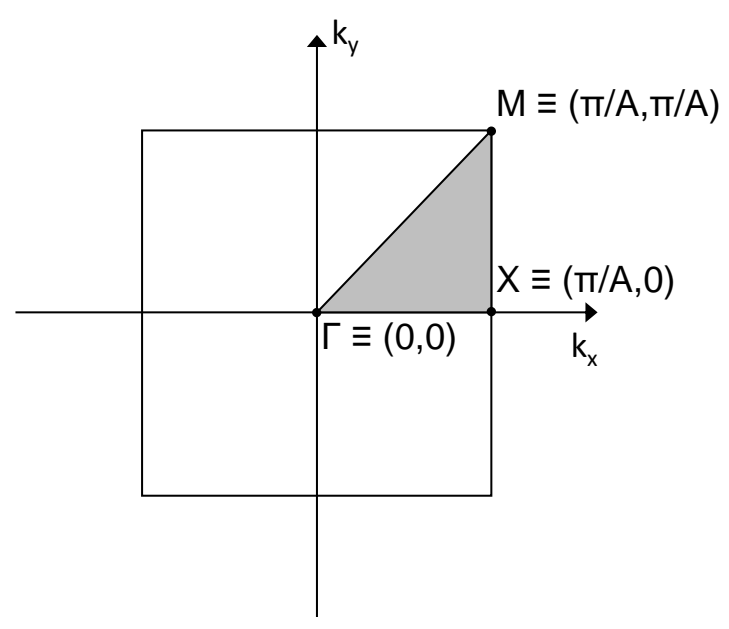

Figure 1: (A) Three-dimensional schematic representation of the unit cell investigated in this study. The structure is obtained by drilling eight cavities arranged in an octagonal pattern in a homogeneous block. The cell is thus divided into three regions, named matrix, ligaments and resonator, respectively. Geometrical parameters are the following: unit cell lattice parameter $A=H=19 \mathrm{~mm}$, internal and external cavity radii $R_{i}=4 t$ and $R_{e}=9 t$, respectively, and ligament thickness $t=1 \mathrm{~mm}$. (B) Schematic representation of the first irreducible Brillouin zone along the which the dispersion curves are calculated. 
A

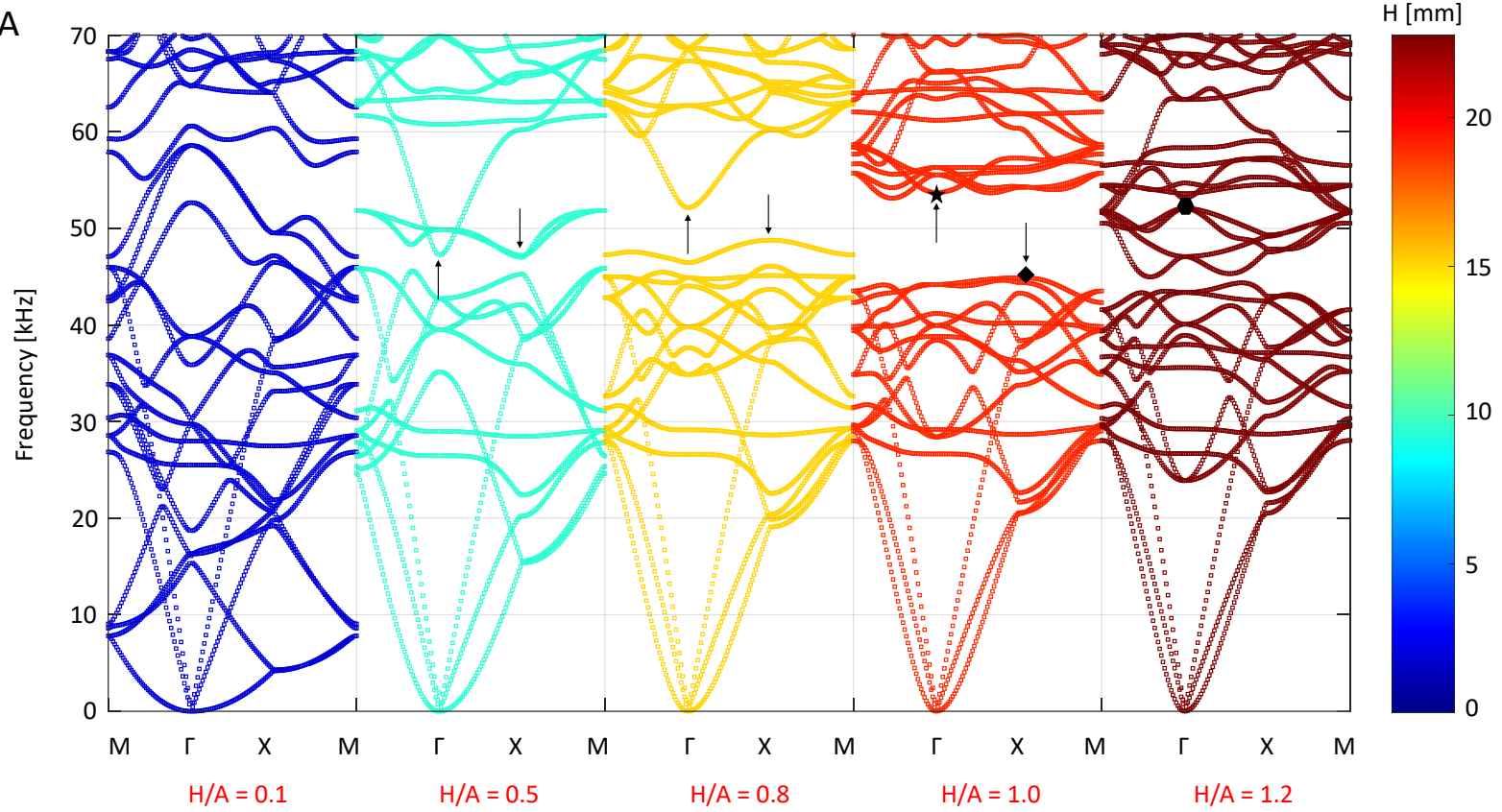

Reduced Wavenumber $\mathrm{k}^{*}$

B

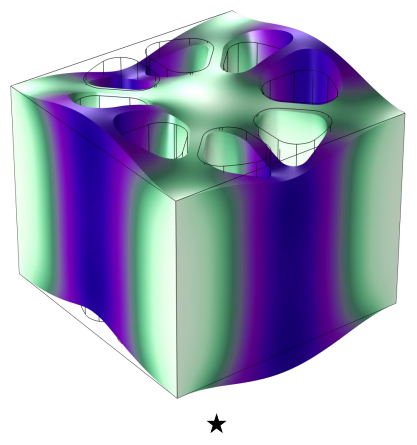

C

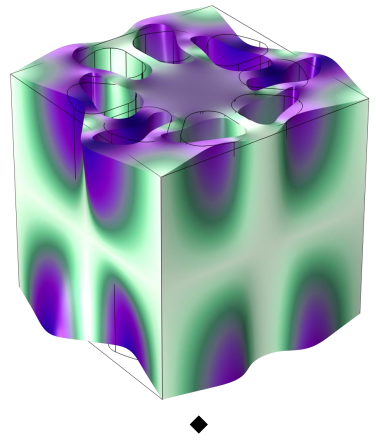

D

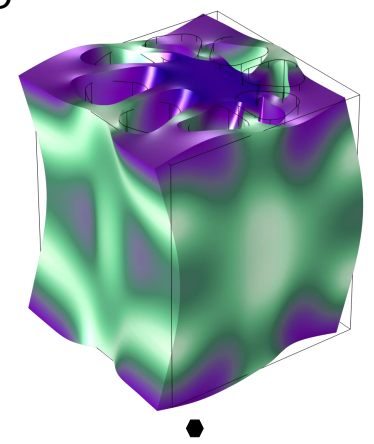

Figure 2: (A) Band diagrams along the $M-\Gamma-X-M$ Brillouin path for the unit cell reported in Fig. 1A presented as a parametric study for different height to lattice parameter ratios $H / A=[0.1,0.5,0.8,1.0,1.2]$. Curves are color coded according to the height $H$ of the unit cell, and range from dark blue (for very thin unit cells) to dark red (for the thicker ones). The influence of the height in the opening of a BG is clearly visible. When the ratio is very small $H / A=0.1$, no $\mathrm{BG}$ is present in the diagram. This is due to the extremely flexible out-of-plane properties of the unit cell, implying a large number of vibration modes in the $[0-70] \mathrm{kHz}$ frequency range. When the height to lattice parameter ratio increases, fewer curves are visible in the diagram and in particular specific modes (highlighted by the black arrows) undergo a frequency shift in opposite directions. This allows to open a BG that increases its width up to a maximum width of $8 \mathrm{kHz}$ achieved when $H / A=1$. If the ratio increases above unity, additional flexural modes tend to reduce the $\mathrm{BG}$ width. (B-D) Deformation of the mode shapes undergoing selective frequency down(up) shift, indicated by a black star and rhombus, and located at the edges of the BG. The additional flexural mode reducing the BG width is also reported as black hexagonal marker 0 These modes are plotted at the $\Gamma$ and $X$ symmetry points. Color map indicates displacement magnitude. 
A

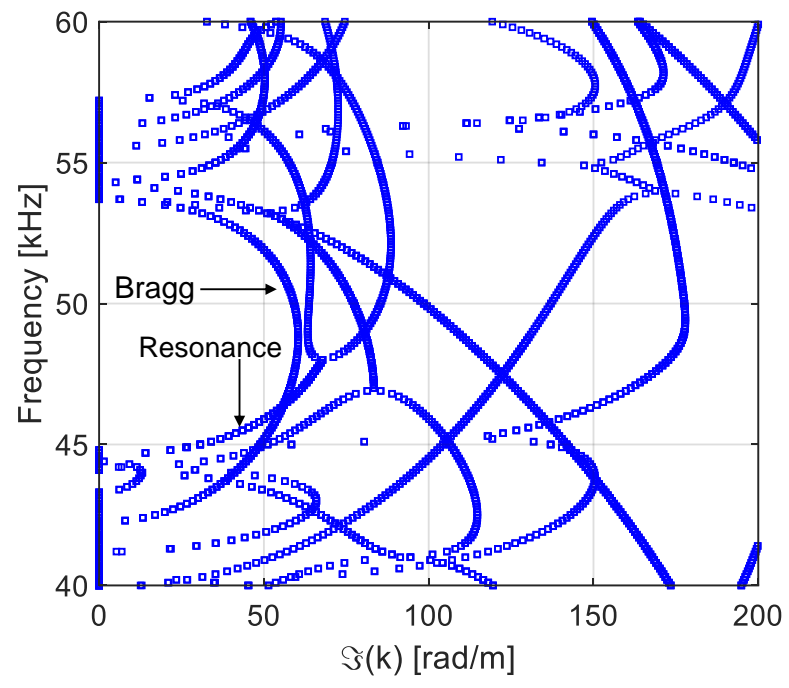

B

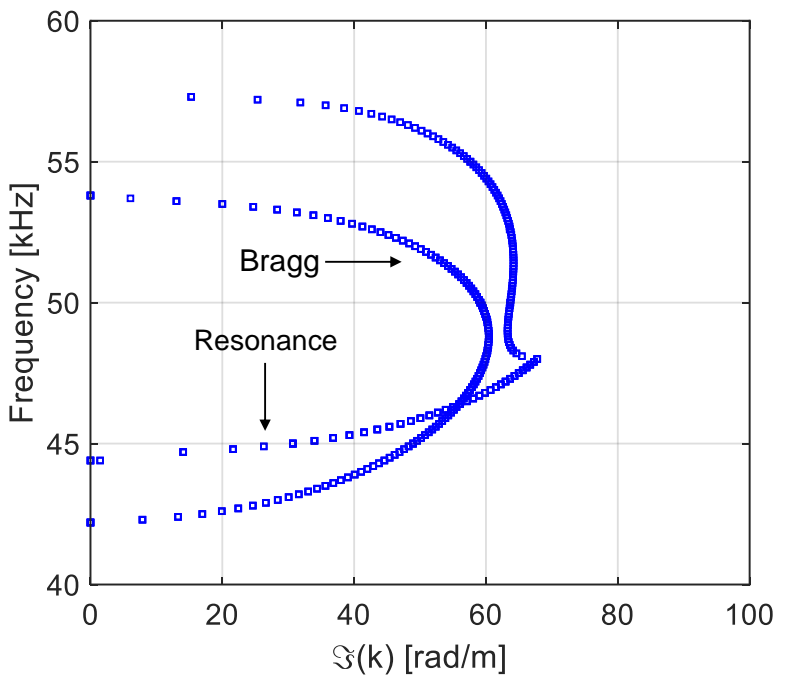

Figure 3: (A) Band diagram along the $\Gamma-X$ path for the $H / A=1$ case reporting the frequency as a function of the imaginary part of the wavenumber, $\varsigma k(\omega)$. (B) Zoom in of the two isolated branches contributing to the mechanism of the BG opening. The typical parabolic and spike-like behaviors of the two curves clearly show how both Bragg and local resonance mechanisms contribute to the BG opening. 

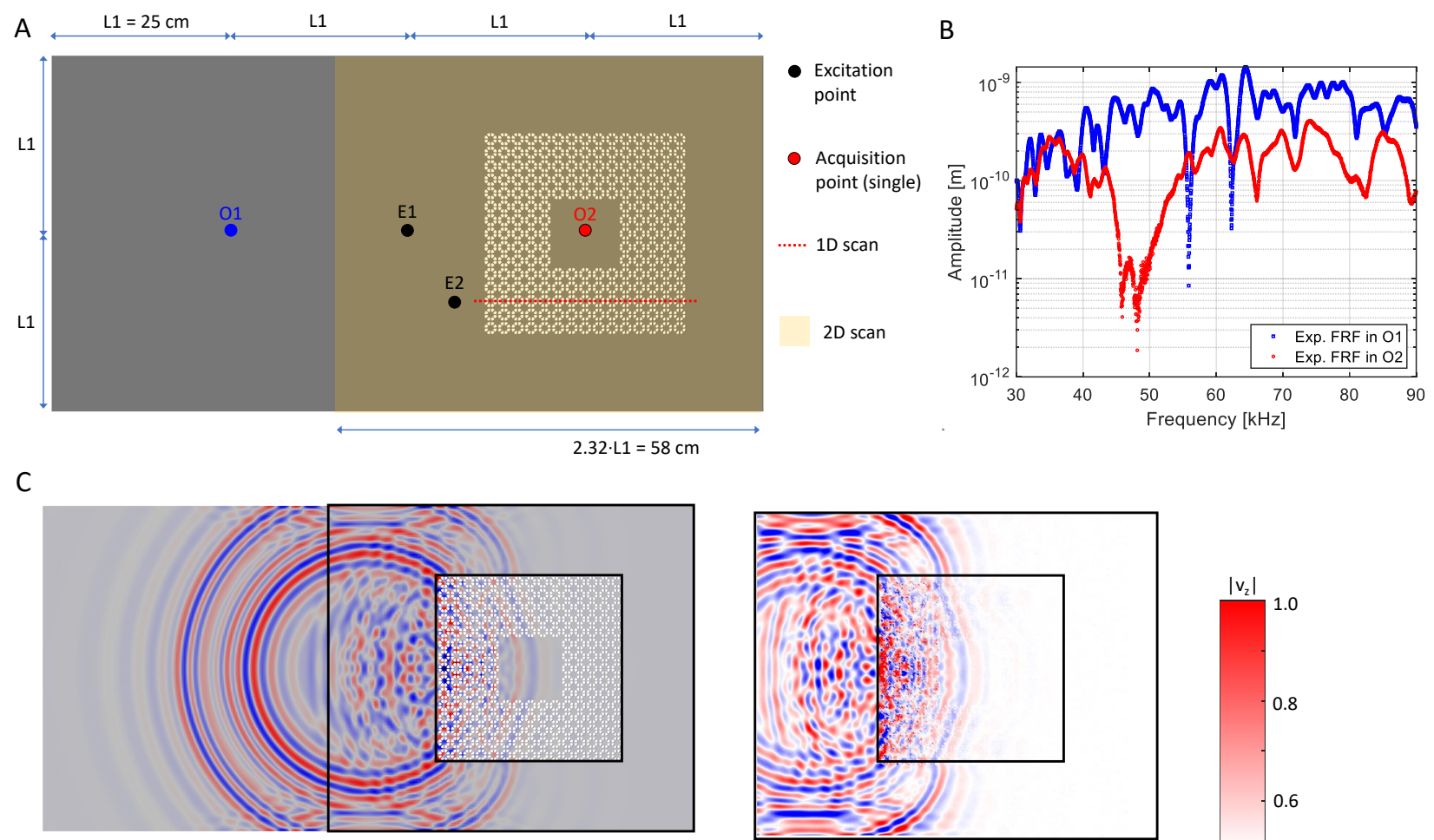

D
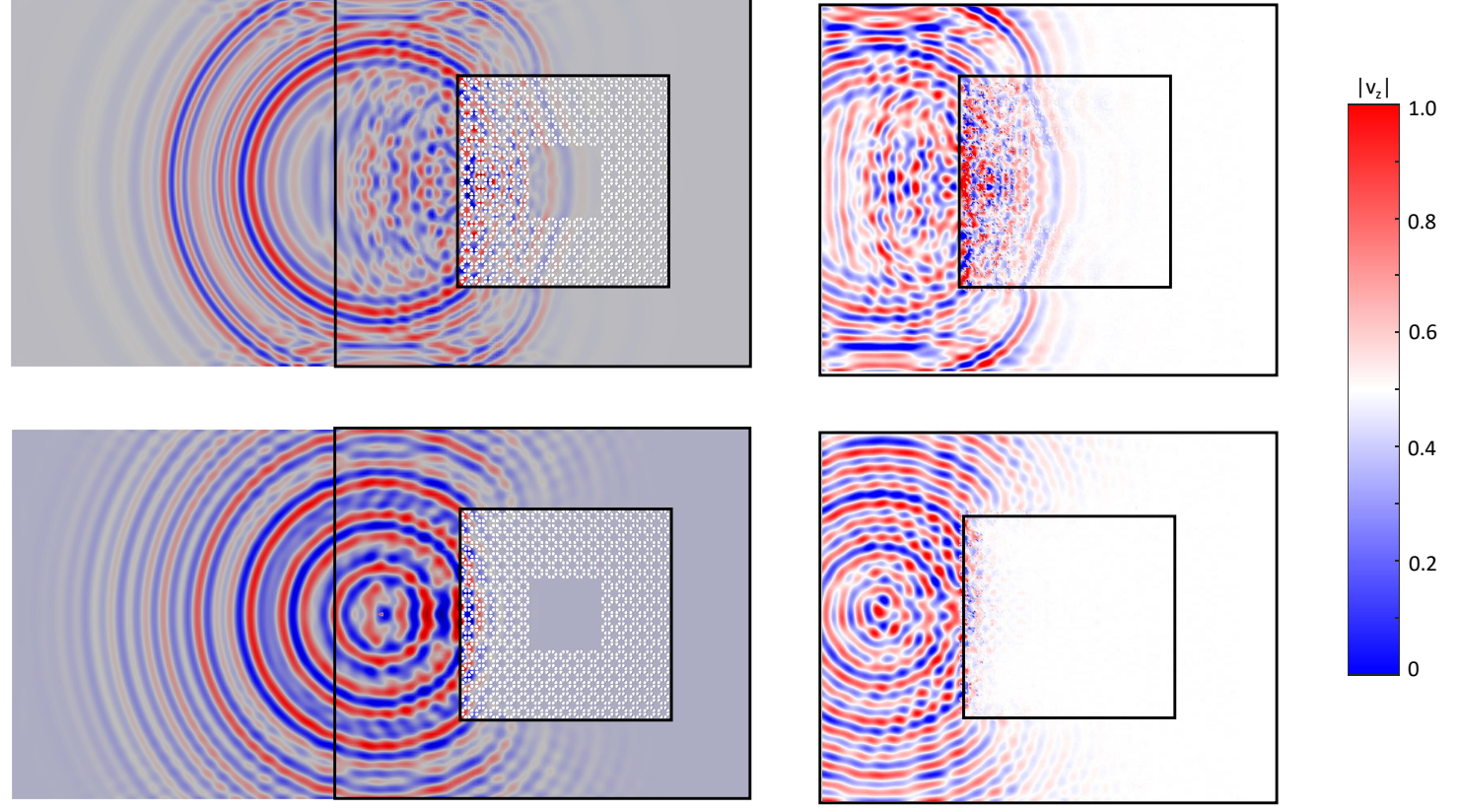

Figure 4: (A) Schematic representation of the FE model used for the three-dimensional transient dynamic computation. The specimen consists of a PMMA rectangular plate of length $4 \cdot L 1=1000 \mathrm{~mm}$, width $2 \cdot L 1=500 \mathrm{~mm}$ and height $H=A=19 \mathrm{~mm}$ ), where 200 unit cells have been drilled in the shape of a square ring. Excitation points are highlighted as black dots. Measurements are performed through Scanning Laser Doppler Vibrometry in specific points outside (blue dot named $O 1$ ) and inside (red dot named $O 2$ ) the phononic ring, along a 1D line scan (dotted red line), and in a 2D region scan (orange rectangle superimposed to the schematics of the plate). (B) Frequency Response Function (FRF) of the system in the $O 1$ and $O 2$ measurement points, both located at $L 1$ from the $E 1$ excitation point. A clear drop in the amplitude is visible in the frequency domain for the measurement inside the phononic region. Numerical (left panel) and experimental (right panel) full wavefield reconstructions of the out-of-plane velocity for a (C) 2 and a (D) 21 sine cycles centered at $50 \mathrm{kHz}$ Hann windowed excitation signals fed in $E 1$. A color map of the out-of plane velocity is reported on the right, and normalized with respect to the maximum displacement. 

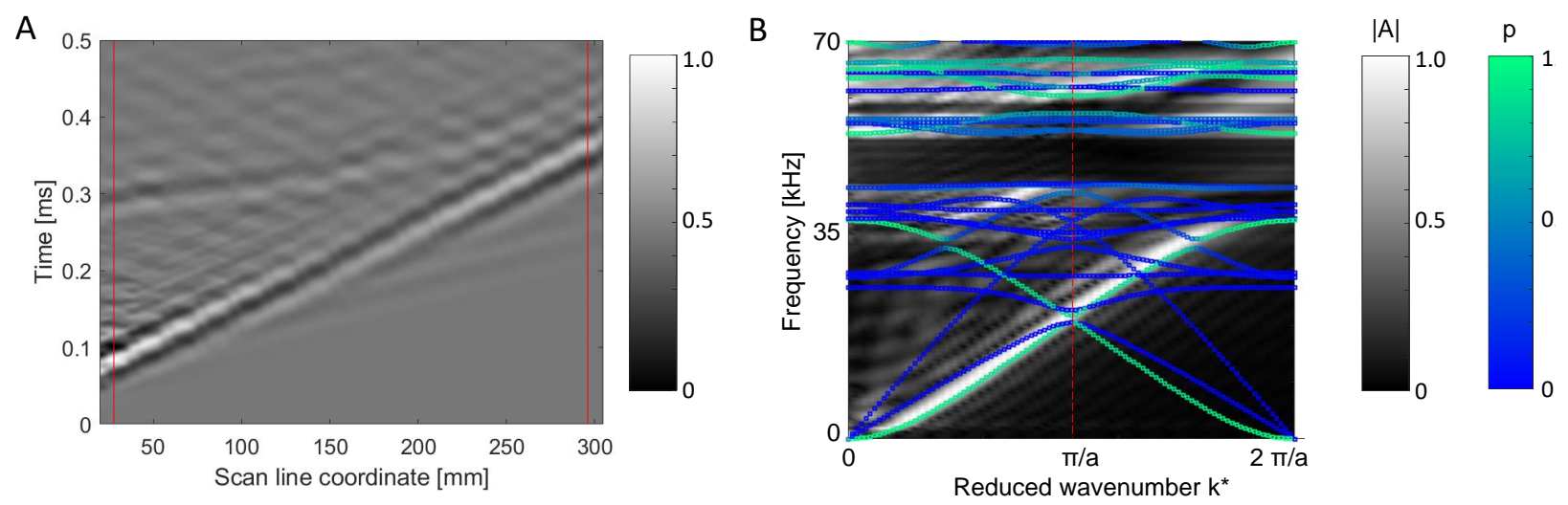

Figure 5: (A) Measured out-of-plane velocity as a function of the scanning position along the dotted red line in Fig. 4 (x-axis) and time (y-axis). Elastic waves are excited at point $E 2$ using a 2 sine cycles centered at $40 \mathrm{kHz}$ and Hann windowed. Red lines denote the beginning and the end of the periodic region. Several reflections due to the impedance mismatch are clearly visible. (B) Wavenumber-frequency representation of the measured signals. Numerical dispersion curves are superimposed to the experimental results. Curves are color-coded depending on their in plane / out-of-plane behavior. Due to the type of experimental set-up, mainly out-of plane modes are excited. 


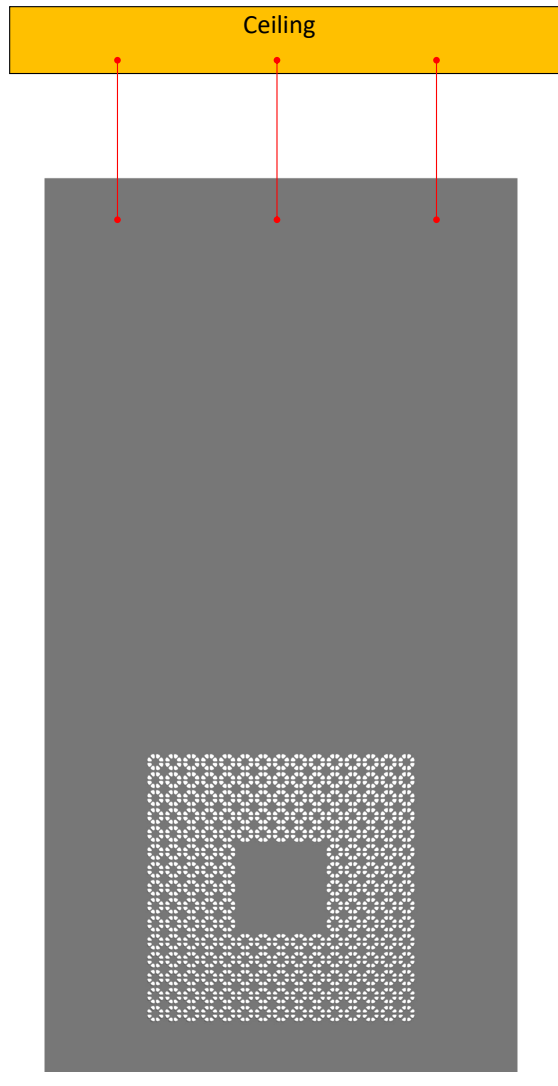

Figure A1: Schematics of vertical hanging of the plate, allowing us to prevent the sample from being subjected to bending, and to mimic the free boundary conditions implemented in the calculations. 


\section{References}

[1] R. Martínez-Sala, J. Sancho, J. V. Sánchez, V. Gómez, J. Llinares, and F. Meseguer. Sound attenuation by sculpture. Nature, 378(6554):241-241, 1995.

[2] P. A. Deymier. Acoustic metamaterials and phononic crystals, volume 173. Springer Science \& Business Media, 2013.

[3] Z. Liu, X. Zhang, Y. Mao, Y. Y. Zhu, Z. Yang, C. T. Chan, and P. Sheng. Locally resonant sonic materials. Science, 289(5485):1734-1736, 2000.

[4] G. Ma and P. Sheng. Acoustic metamaterials: From local resonances to broad horizons. Science advances, 2(2):e1501595, 2016.

[5] J. O. Vasseur, P. A. Deymier, B. Chenni, B. Djafari-Rouhani, L. Dobrzynski, and D. Prevost. Experimental and theoretical evidence for the existence of absolute acoustic band gaps in two-dimensional solid phononic crystals. Physical Review Letters, 86(14):3012, 2001.

[6] M. Miniaci, A. Marzani, N. Testoni, and L. De Marchi. Complete band gaps in a polyvinyl chloride (pvc) phononic plate with cross-like holes: numerical design and experimental verification. Ultrasonics, 56:251-259, 2015.

[7] B. Morvan, A. Tinel, A.-C. Hladky-Hennion, J. O. Vasseur, and B. Dubus. Experimental demonstration of the negative refraction of a transverse elastic wave in a two-dimensional solid phononic crystal. Applied Physics Letters, 96(10):101905, 2010.

[8] J. Pierre, O. Boyko, L. Belliard, J. O. Vasseur, and B. Bonello. Negative refraction of zero order flexural lamb waves through a two-dimensional phononic crystal. Applied Physics Letters, 97(12):121919, 2010.

[9] V. M. García-Chocano, J. Christensen, and J. Sánchez-Dehesa. Negative refraction and energy funneling by hyperbolic materials: An experimental demonstration in acoustics. Physical review letters, 112(14):144301, 2014. 
[10] D. Bigoni, S. Guenneau, A. B. Movchan, and M. Brun. Elastic metamaterials with inertial locally resonant structures: Application to lensing and localization. Physical Review B, 87(17):174303, 2013.

[11] A. Khelif, M. Wilm, V. Laude, S. Ballandras, and B. Djafari-Rouhani. Guided elastic waves along a rod defect of a two-dimensional phononic crystal. Physical Review E, 69(6):067601, 2004.

[12] G. Bordiga, L. Cabras, D. Bigoni, and A. Piccolroaz. Free and forced wave propagation in a rayleigh-beam grid: flat bands, dirac cones, and vibration localization vs isotropization. International Journal of Solids and Structures, 161:64-81, 2019.

[13] S. H. Mousavi, A. B. Khanikaev, and Z. Wang. Topologically protected elastic waves in phononic metamaterials. Nature communications, 6(1):1-7, 2015.

[14] R. K. Pal and M. Ruzzene. Edge waves in plates with resonators: an elastic analogue of the quantum valley hall effect. New Journal of Physics, 19(2):025001, 2017.

[15] M. Miniaci, R. K Pal, B. Morvan, and M. Ruzzene. Experimental observation of topologically protected helical edge modes in patterned elastic plates. Physical Review X, 8(3):031074, 2018.

[16] M. Miniaci, R. K. Pal, R. Manna, and M. Ruzzene. Valley-based splitting of topologically protected helical waves in elastic plates. Physical Review B, 100(2):024304, 2019.

[17] C.-W. Chen, N. Lera, R. Chaunsali, D. Torrent, J. V. Alvarez, J. Yang, P. San-Jose, and J. Christensen. Mechanical analogue of a majorana bound state. Advanced Materials, 31(51):1904386, 2019.

[18] G. Ma, C. Fu, G. Wang, P. Del Hougne, J. Christensen, Y. Lai, and P. Sheng. Polarization bandgaps and fluid-like elasticity in fully solid elastic metamaterials. Nature communications, 7(1):1-8, 2016.

[19] M. Gei, A. B. Movchan, and D. Bigoni. Band-gap shift and defect-induced annihilation in prestressed elastic structures. Journal of Applied Physics, 105(6):063507, 2009. 
[20] R. V. Craster and S. Guenneau. Acoustic metamaterials: Negative refraction, imaging, lensing and cloaking, volume 166. Springer Science \& Business Media, 2012.

[21] E. Baravelli and M. Ruzzene. Internally resonating lattices for bandgap generation and low-frequency vibration control. Journal of Sound and Vibration, 332(25):6562-6579, 2013.

[22] M. .I Hussein, M. J. Leamy, and M. Ruzzene. Dynamics of phononic materials and structures: Historical origins, recent progress, and future outlook. Applied Mechanics Reviews, 66(4), 2014.

[23] S. Taniker and C. Yilmaz. Design, analysis and experimental investigation of threedimensional structures with inertial amplification induced vibration stop bands. International Journal of Solids and Structures, 72:88-97, 2015.

[24] M. Mazzotti, M. Miniaci, and I. Bartoli. Band structure analysis of leaky bloch waves in 2d phononic crystal plates. Ultrasonics, 74:140-143, 2017.

[25] M. Mazzotti, I. Bartoli, and M. Miniaci. Modeling bloch waves in prestressed phononic crystal plates. Frontiers in Materials, 6:74, 2019.

[26] C. Sugino, M. Ruzzene, and A. Erturk. Merging mechanical and electromechanical bandgaps in locally resonant metamaterials and metastructures. Journal of the Mechanics and Physics of Solids, 116:323-333, 2018.

[27] A. Bergamini, M. Miniaci, T. Delpero, D. Tallarico, B. Van Damme, G. Hannema, I. Leibacher, and A. Zemp. Tacticity in chiral phononic crystals. Nature communications, 10(1):1-8, 2019.

[28] M. Miniaci, A. S. Gliozzi, B. Morvan, A. Krushynska, F. Bosia, M. Scalerandi, and N. M. Pugno. Proof of concept for an ultrasensitive technique to detect and localize sources of elastic nonlinearity using phononic crystals. Physical review letters, 118(21):214301, 2017.

[29] F. Ciampa, A. Mankar, and A. Marini. Phononic crystal waveguide transducers for nonlinear elastic wave sensing. Scientific reports, 7(1):1-8, 2017. 
[30] A. S. Gliozzi, M. Miniaci, A. Chiappone, A. Bergamini, B. Morin, and E. Descrovi. Tunable photo-responsive elastic metamaterials. Nature communications, 11(1):1-8, 2020.

[31] B. Rostami-Dogolsara, M. K. Moravvej-Farshi, and F. Nazari. Designing switchable phononic crystal-based acoustic demultiplexer. IEEE transactions on ultrasonics, ferroelectrics, and frequency control, 63(9):1468-1473, 2016.

[32] R. Ardito, M. Cremonesi, L. D'Alessandro, and A. Frangi. Application of optimallyshaped phononic crystals to reduce anchor losses of mems resonators. In 2016 IEEE International Ultrasonics Symposium (IUS), pages 1-3. IEEE, 2016.

[33] D. Misseroni, D. J. Colquitt, A. B. Movchan, N. V. Movchan, and I. . Jones. Cymatics for the cloaking of flexural vibrations in a structured plate. Scientific reports, 6:23929, 2016.

[34] M. Miniaci, M. Mazzotti, M. Radzieński, N. Kherraz, P. Kudela, W. Ostachowicz, B. Morvan, F. Bosia, and N. M. Pugno. Experimental observation of a large low-frequency band gap in a polymer waveguide. Frontiers in Materials, 5:8, 2018.

[35] J.-H. Sun and T.-T. Wu. Propagation of acoustic waves in phononic-crystal plates and waveguides using a finite-difference time-domain method. Physical Review B, 76(10):104304, 2007.

[36] Y. Pennec, J. O. Vasseur, B. Djafari-Rouhani, L. Dobrzyński, and P. A. Deymier. Twodimensional phononic crystals: Examples and applications. Surface Science Reports, 65(8):229-291, 2010.

[37] Y. Yao, F. Wu, Z. Hou, and Z. Xin. Lamb waves in two-dimensional phononic crystal plate with anisotropic inclusions. Ultrasonics, 51(5):602-605, 2011.

[38] M. Rupin, F. Lemoult, G. Lerosey, and P. Roux. Experimental demonstration of ordered and disordered multiresonant metamaterials for lamb waves. Physical review letters, 112(23):234301, 2014

[39] Y. Jin, B. Bonello, R. P. Moiseyenko, Y. Pennec, O. Boyko, and B. Djafari-Rouhani. Pillar-type acoustic metasurface. Physical Review B, 96(10):104311, 2017. 
[40] M. Bavencoffe, A.-C. Hladky-Hennion, B. Morvan, and J.-L. Izbicki. Attenuation of lamb waves in the vicinity of a forbidden band in a phononic crystal. IEEE transactions on ultrasonics, ferroelectrics, and frequency control, 56(9):1960-1967, 2009.

[41] L. De Marchi, A. Marzani, and M. Miniaci. A dispersion compensation procedure to extend pulse-echo defects location to irregular waveguides. NDT \& E International, $54: 115-122,2013$.

[42] M. Collet, M. Ouisse, M. Ruzzene, and M. N. Ichchou. Floquetâ€"bloch decomposition for the computation of dispersion of two-dimensional periodic, damped mechanical systems. International Journal of Solids and Structures, 48(20):2837 - 2848, 2011.

[43] W. Ostachowicz, P. Kudela, M. Krawczuk, and A. Zak. Guided Waves in Structures for SHM: The Time - domain Spectral Element Method. A John Wiley \& Sons, Ltd., publication. Wiley, 2012.

[44] M. Miniaci, M. Mazzotti, A. Amendola, and F. Fraternali. Effect of prestress on phononic band gaps induced by inertial amplification. International Journal of Solids and Structures, 2021.

[45] P. Kudela, M. Radzieński, and W. Ostachowicz. Identification of cracks in thin-walled structures by means of wavenumber filtering. Mechanical Systems and Signal Processing, 50:456-466, 2015. 